\title{
ETHICAL ISSUES IN EPIDEMIOLOGICAL STUDIES
}

\author{
Chirom Ranjeev Kumar ${ }^{1}$, Thokchom Shantajit ${ }^{1}$, Abhay Gaidhane ${ }^{2}$ \\ ${ }^{1}$ Resident, ${ }^{2}$ Professor \& Head, Department of Community Medicine, Jawaharlal Nehru Medical College, \\ Datta Meghe Institute of Medical Sciences, Sawangi Meghe, Wardha, Maharashtra, India.
}

\section{ABSTRACT}

Epidemiology is the study of the distribution and determinants of health related states and events in populations, and its application to the control of health problems. An epidemiological studies involve the population and as such ethical issues are an important aspect of it which need to be properly understood and adhered to. These issues can relate to informed consent, appropriate sample size, selection of participants, confidentiality, conflict of interest etc. Studies like therapeutic trial have also a lot of ethical issues which differ from issues pertaining to non-therapeutic trials. The source of funding for the study can have implication. Fabrication of data, manipulation during analysis, intentional changes in the result to obtain a pre desired outcome are some grey areas which need to be addressed. Another ethical issue is publication in certain Journals with monetary involvement. Lastly but not the least, authorship also have certain ethical issues. Attention to ethical issues can facilitate the effective planning, implementation, and growth of a variety of public health programs and research activities. Moreover with advancement in Epidemiology like molecular epidemiology, genetic epidemiology etc. the ethical issues become more complicated.

KEYWORDS: Ethics; Epidemiology; Epidemiological studies; Ethical Issues.

\section{INTRODUCTION}

Epidemiology is the study of the distribution and determinants of health related states and events in populations, and its application to the control of health problems [1]. As such majority of the epidemiological studies, either descriptive or analytical involves the population, and population are composed of individuals. Therefore interacting with the individuals during the course of a study requires that ethical issues need to be addressed. Epidemiology has contributed to the improvement in human health achieved over the past century. It is expected that the field will continue to do so by using ever more powerful and sophisticated scientific tools that are being developed endlessly. This will help in increasing understanding of the distribution of health and illness and the related determinants of health which includes physical, chemical, biological, behavioural, social and environmental determinants [2].

Epidemiological studies can include a wide range of research like investigation of disease causation, to the evaluation of health services and programs, to largescale studies of new public health interventions to pre-

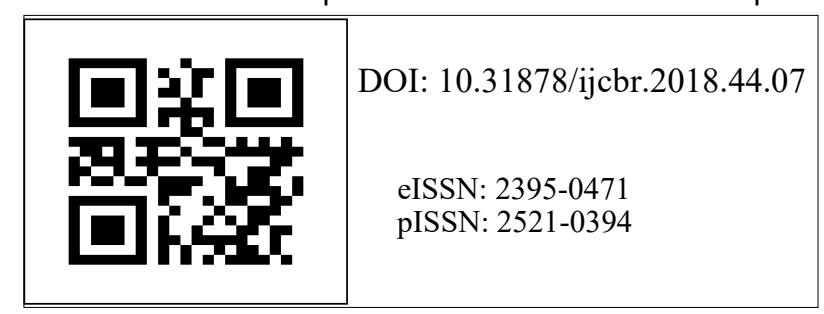

vent disease(2). Morever, epidemiological studies can either be therapeutics or non-therapeutics, and this has implication on ethical issues involved which vary depending on the type of the research.

Minimizing risks and potential harms and maximizing potential benefits are particularly important in epidemiologic studies of vulnerable populations. These vulnerable populations include geriatric people, people living with HIV (PLHIV), slum residence and migrant workers [3].

In order to have a better insight into the different ethical issues that arises in Epidemiological studies, the Study can be subdivided into different stages starting from conception of ideas for undertaking it up to the publication of the research and the different ethical issues involved in these stages.

Planning an Epidemiological research: Formulating a research topic should focused on the health and welfare of the population and should not be based on feasibility alone. Selection is of paramount importance as the conclusion of the research should benefit the population. Ethics demand that areas where thrust are needed, as determined by existing literature on that aspect, and of priority importance to the public, should be emphasized upon. All proposals to conduct epidemiological studies involving human subjects should be cleared by one or more scientific review and ethical review committees $[2,4]$.

Corresponding author: Dr. Chirom Ranjeev Kumar, Resident, Department of Community Medicine, Jawaharlal Nehru Medical College, Datta Meghe Institute of Medical Sciences, Sawangi Meghe, Wardha, Maharashtra, India.

Email: chirom.ranjeev@gmail.com

International Journal of Clinical and Biomedical Research. (C) 2018 Sumathi Publications.

This is an Open Access article which permits unrestricted non-commercial use, provided the original work is properly cited. 
Study design: The study design should be proper for the research. It should be addressed specifically in the research protocol. Wherever needed, the clearance from the Institutional Ethical Committee or the relevant competent authority should be obtained.

The design should be strictly adhered to throughout the completion of the study [4].

\section{Conduct of the study}

Setting of the study should commensurate with research question that is needed to be answered. Sample size and selection should adhere to standard protocol. The sample should be representative of the population so that it can be generalizable.

Certain researcher have a tendency to exclude certain areas to make their research more easy and feasible and, it thus decreases the generalizability and is unethical.

2. Informed consent is another important aspect in Epidemiological Research. It is important to explain the procedures carefully and preferably in local vernacular, keeping in mind local customs and traditions. The risk and hazards of any procedure if involve in the study should be properly explained, and every effort should be made so that the expected participants clearly understand it. The purpose of the research, any scientific procedure involved, any inconveniences or discomfort that can arise should also be explained to the expected participants. After this, the participants right to refusal for participation or withdraw at any time should be respected. This is an important ethical aspect. It should be kept in mind that, there are certain research where Informed consent need not be taken. It is virtually impossible to obtained informed consent in researches that involve data from certain International, National or Regional Surveys $[3,5,6]$.

\section{Confidentiality}

Confidentiality of any data obtained should be guaranteed. It should be explicitly explained to the participants. During the course of the study, if any participants are found be having any problems, medical illnesses, proper mechanism should be made so that the participants get a forum for redressal of it. The study in order to test the hypothesis required that relevant information are collected from selected persons from a population and these information, if disclosed to third parties, may cause harm or distress $[7,8]$.

\section{Analysis}

Plan for analysis of the study should be meticulously develop ahead before the study commence based on the distribution and characteristic of the data likely to be obtained. This will prevent manipulation of data later on. Data manipulation usually occur when the data obtained are not conforming to the desired result and this is an ethical challenge. Researchers are not always honest. Fabrication of data are done to arrive at selective results so as to validate the hypothesis being pursued and at the same time certain valid results contradictory to it are omitted. Sometime intentional changes are made in the analysis to obtain a pre desired outcome [4].

\section{Publication}

Irrespective of the outcome of the research that is carried out, it should be made known to the scientific community. Shortcomings and limitation should be clearly spelt out. Certain researchers have a tendency to publish their result in certain Journals which do not adhere to a strict rule but set up for monetary gains $[4,5]$.

Conflict of Interest and Sponsorship should be clearly spelt out. Similarly authorship should be clearly spelled out. There is a tendency to add certain person who are not involve in the research and this is an unethical issue. Only those who have contributed to the study should be given due credit $[4,7,9]$.

With the advancement of Epidemiology, like molecular epidemiology, genetic epidemiology etc. studies pertaining to these field involve more complex ethical issues. Ethical guidelines related to it as per the regulation of the country or region should be complied with in these studies [10].

\section{CONCLUSION}

Epidemiological studies involve population and as such individuals. These studies are a part of medical Science based on scientific facts. Well prepared Studies yield results which can be generalized for the population and these can benefit the public. By its nature and character, ethical issues are involved in every aspect of epidemiological studies. These issues start from the very formulation of the studies till its culmination into result. To generate valid scientific knowledge, facts ethical consideration need to be accounted for. Ethical principles in respect of autonomy, non-maleficence, beneficence and justice need to be adhered to. Sincerity, integrity, transparency should be guiding principle.

\section{REFERENCES}

1) Basic epidemiology $2^{\text {nd }}$ edition by Bonita R, Beaglehole $212 \mathrm{p}$.

2) International Ethical Guidelines for Epidemiological Studies,CIOMS,WHO, pdf

3) Coughlin SS. Ethical issues in epidemiologic research and public health practice. Emerg Themes Epidemiol. 2006;10.

4) Hessel PA, Fourie PB. Ethical issues in epidemiologica research. :4. 
5) Ethics Training Manual.pdf. ETHICS TRAINING RESOURCE GROUP, INDIA. 2007.

6) Bánkowski Z, Bryant JH, Last JM, Council for International Organizations of Medical Sciences, editors. Ethics and epidemiology: international guidelines: proceedings of the XXVth CIOMS Conference, Geneva, Switzerland, 7-9 November 1990. Geneva: CIOMS; 1991. 163 p.

7) Coughlin SS. Ethical issues in epidemiologic research and public health practice. Emerg Themes Epidemiol. 2006;10.

8) University of Miami, Department of Epidemiology and Public Health, EPH 525-GY pdf

9) Waters WE. Ethics and epidemiological research. Int J Epidemiol. 1985;14(1):48-51.

10) Holtzman NA, Andrews LB. Ethical and Legal Issues in Genetic Epidemiology. Epidemiol Rev. 1997 Jan 1;19(1):163-74.

How to Cite this article: Chirom Ranjeev Kumar, Thokchom Shantajit, Abhay Gaidhane. Ethical issues in epidemiological studies. Int. j. clin. biomed. res. 2018;4(4): 33-35. 